\title{
Utilization of 3D sensors in robotics
}

\author{
Miroslav Marcaník ${ }^{1, *}$, Michal Šustek ${ }^{1}$, Pavel Tomášek ${ }^{1}$, and Roman Jašek ${ }^{1}$ \\ ${ }^{1}$ Tomas Bata University in Zlín, Faculty of Applied Informatics, Nad Stráněmi 4511, 760 05, Zlín, Czech Republic
}

\begin{abstract}
Recently, the interest in development of service robots highly increases. The annual turnover in this area is estimated to a number close to 17 milliard Euro in the past years. The annual turnover could rise to 0.1 billion Euro by 2020 by IFR considering $30 \%$ growth every year. Investments are expected to flow into all areas related to service robotics, mainly into the development of assistant robots for seniors and the handicapped people, health monitoring and surgical robots, robots in agriculture, pilotless drones and helicopters and ground vehicles without a driver. Very promising years seems to be coming for all the new and already existing companies focused on this area with their software and sensor engineers together with producers of important accessories.
\end{abstract}

\section{Introduction}

Monitoring is used in various non-engineering applications. Tasks not only of this type are operated by service robots all over the world. Robots are equipped with a camera system for these purposes. The data from cameras are recorded and transmitted to the standpoint of an operator. Service robots can be favourably utilized wherever the environment is dangerous for a man or difficult to reach. It could be for example searching for a dangerous material, security purposes, monitoring complicated areas, undersea research.

Basic terms related to robotics, taxonomy, classification of robots and examples of complex robots are presented in the following section. The next section is focused on utilization of sensors, sensorial systems and how they work. In the last section the principle of 3D scanning of objects in free space is described together with an application in a real project.

\section{Robotics}

Robotics is a modern multidisciplinary field including mechanics, electronics, control theory, measurement techniques, artificial intelligence and other disciplines. Currently, robotics is mainly connected with the area of automatization. The first appliance called as a robot was produced at the beginning of the twentieth century. The word of robot is known already since 1920. Čapek brothers (Czech writers) created this word in their book with the name of R.U.R. A robot represents a nonbiological artificial imitation a man [1].

\subsection{Classification of robots}

The robots can be divided by several different aspects.

Dividing by mobility:
- Stationary - they cannot move from one place to another (manipulators in the industry, for instance),

- Mobile - they are able to move (satellites or vehicles in the aerospace).

Mobile robots can be divided by several other criteria [2]:

- Remotely controlled - they are controlled by an operator, who has a visual information about the space around such a robot,

- Autonomous - they are supposed to solve tasks independently, for instance line tracking, ability to react on obstructions in their way, ability to move in an unknown area.

Moreover, robots can be divided by the environment in which will the robot operate:

- On the land - wheel and full-track vehicles.

- In the water - explorational submarines, robotic fish,

- In the air - quadcopters, drones, RC models, robotic birds,

- In the aerospace (outer space) - service robots, satellites.

Dividing by the purpose:

- Manipulatory - for manipulations with objects,

- Assembly - as a part of complex assembling lines,

- Service - for repairs and maintenance at places out of simple reach,

- Inspectional - for controlling of a state of an observed phenomenon,

- Explorational - for exploring of an unknown area,

- Military - pyrotechnical robots, drones,

- Medical - manipulators, laser scalpels,

- For fun - quadcopters, drones, RC models, Dividing by the type of a running gear:

\footnotetext{
* Corresponding author: marcanik@fai.utb.cz
} 
- Wheel,

- Full-track,

- Walking,

- Crawling,

- Climbing,

- Hybrid,

- Intelligent.

\subsection{Examples of robots}

ASIMO - Honda (mobile - autonomous - for walking on the land - medical aims): It is probably the best publicly known robot. It was introduced in 2000. ASIMO was constructed to imitate a human being and thus to replace him in a common human interaction. Therefore this is a humanoid. ASIMO could server as companion for elderly or ill people. It can react to a human behaviour and speech. It is not applicable in exacting environment or a terrain (compared to robots from Boston Dynamics). ASIMO is $130 \mathrm{~cm}$ high. It weighs $50 \mathrm{~kg}$. Its maximal speed is $5.7 \mathrm{~km} / \mathrm{h}, 2.7 \mathrm{~km} / \mathrm{h}$ normally. ASIMO has 57 degrees of freedom (DOF) totally. What is very interesting about ASIMO is the fact that ASIMO can be controlled by a special head cap. Thoughts (electrical charges) are captured by sensors on the cap and then the cap transmits the signals. These signals can control the movement of the robot. Overall, operation of such a robot is very energetically exhaustive because there is a need of an actuator for every DOF. Therefore it can operate only one hour on its internal battery [2].

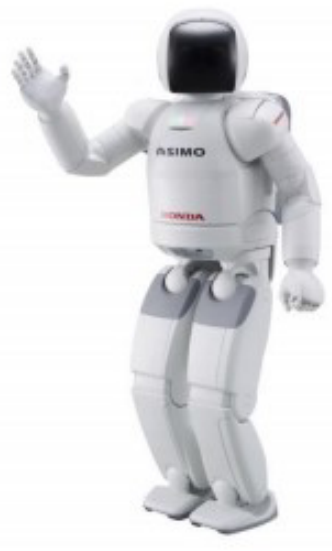

Fig. 1. ASIMO [2].

Mini AERCam - NASA (mobile - autonomous - for outer space - flying - inspectional/service): This robotic camera was developed by NASA in 2005 for inspections in the outer space. It is a freely flying robot equipped with 12 nozzles for compressed gas allowing movements and rotations in all directions. It is able to orientate in complex three dimensional space autonomously. It has a spherical shape. The diameter is $190 \mathrm{~mm}$ and it weighs $4.5 \mathrm{~kg}[2]$.
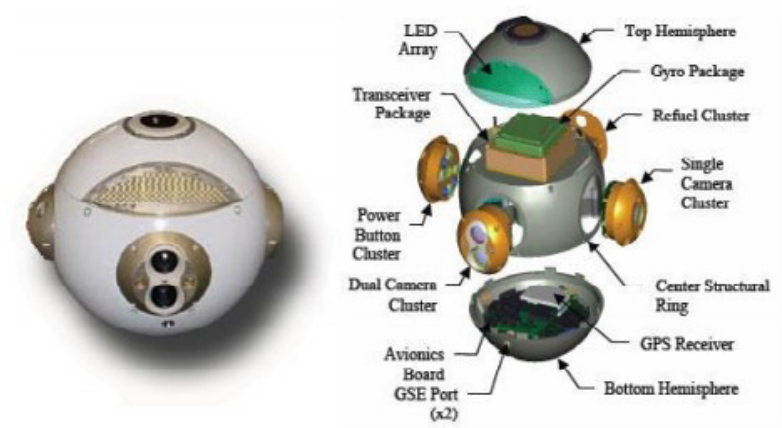

Fig. 2. Mini AERCam [2].

ATLAS - Boston Dynamics (mobile - autonomous - on the land - walking - multifunctional): Atlas is highly mobile humanoid specially constructed for exacting terrain. It was developed by Boston Dynamics with the support of Defence Advanced Research Projects Agency (DARPA). It can climg, run, manipulate and interact with a surrounding environment. It is $180 \mathrm{~cm}$ high. It was introduced in 2013. Its arms contain joints which allows ATLAS to use common human tools in its hands. Atlas is prepared to work in dangerous areas in which a man cannot survive. Therefore ATLAS is able to use instruments, manipulate, work with electrical appliances and perform many other similar tasks. It is equipped with a high number of sensors, stereo camera, and laser range-finder. It has implemented 28 DOF. ATLAS operates on a cable connected to an external power source [2].

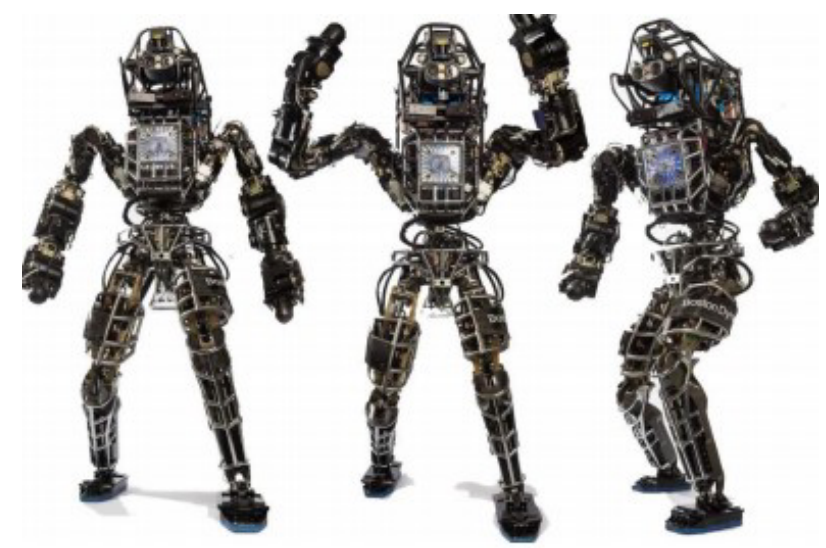

Fig. 3. ATLAS [2].

An Unmanned Aerial Vehicle (UAV) or also a drone is a plane without crew which can be controlled remotely or fly autonomously using programmed flying plans or using more complex dynamic autonomous systems. These aeroplanes have a common military utilization. They can explore and also attack. They can also serve in some specific civil tasks (for example in fire fighting, monitoring used by police or for exploration of some terrain) [3]. 


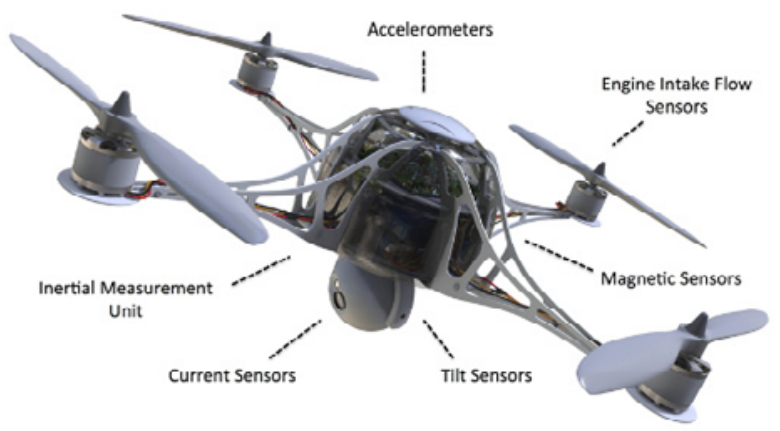

Fig. 4. Model UAV [3].

\section{Sensors}

Sensors are elements, which produce input block measuring chain, then this chain is in direct contact with the measured medium. Other labels of sensors are for example sensor, detector, converter, and scanner [4].

Sensors could be ordered by usability into the following categories:

- For measuring quantities,

- For physical principles,

- For the measurement environment,

- For signals according to their transformation,

- For Manufacturing Technology.

Changes of measuring quantities are scan over sensitive element mostly known as receptor, then subsequent evaluation in the sensor circuit (analog/digital converter), where the measured value is converted mostly into electric impulse, which could be processed or could be used directly as the output quantity. Principle of sensor is shown at Figure 5.

\section{Input}

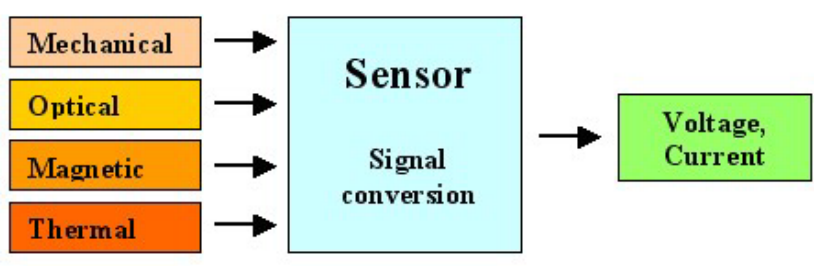

Fig. 5. Principle of a sensor [6].

First smart sensor (1978) Beckenbridge and Husson, was defined as: Smart sensor contains functions for processing measured data, automatic correction measured data, and it could automatically detect and eliminate abnormal and wrong values. It also contains a set of algorithms, which allows to respond on changes of external conditions.

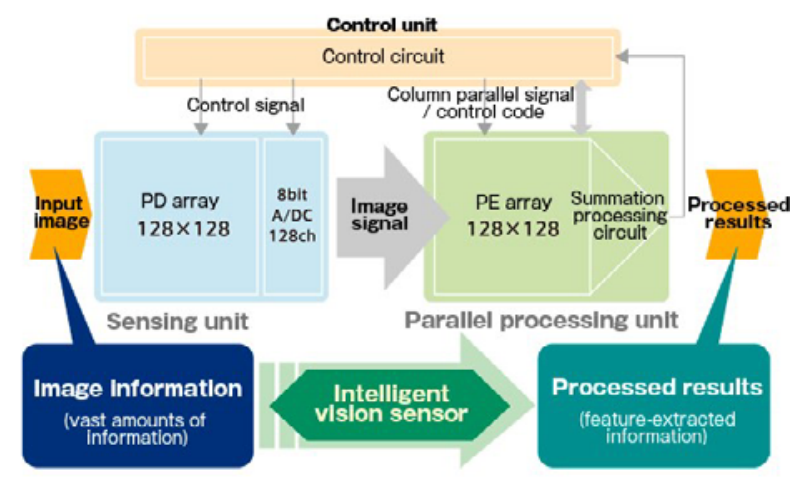

Fig. 6. The principle of an intelligent sensors [6].

3D sensor can be defined as a measuring unit which senses an object in 3D space or occupies angles $\mathrm{X}$, Y and Z. 3D sensors operate on the principle of two or more video cameras, or for engaging the third dimension may be used most of the laser scanner. This makes possible a whole or only a certain part transform into 3D and 3D scene then analyses and selects the best way to handle [5].

From the above description 3D sensors can be divided into two basic categories by using various sensors. They are active and passive sensing:

- Active scanning uses laser/camera, additional information or other auxiliary devices. The concept of active sensing is very closely linked to the concept of "creating depth map".

- Passive versus active scan uses only one sensor the camera/laser.

Since it is an improved sensor it can be categorized in a similar $3 \mathrm{D}$ sensors. A general allocation can be seen in Fig. 6 [5].

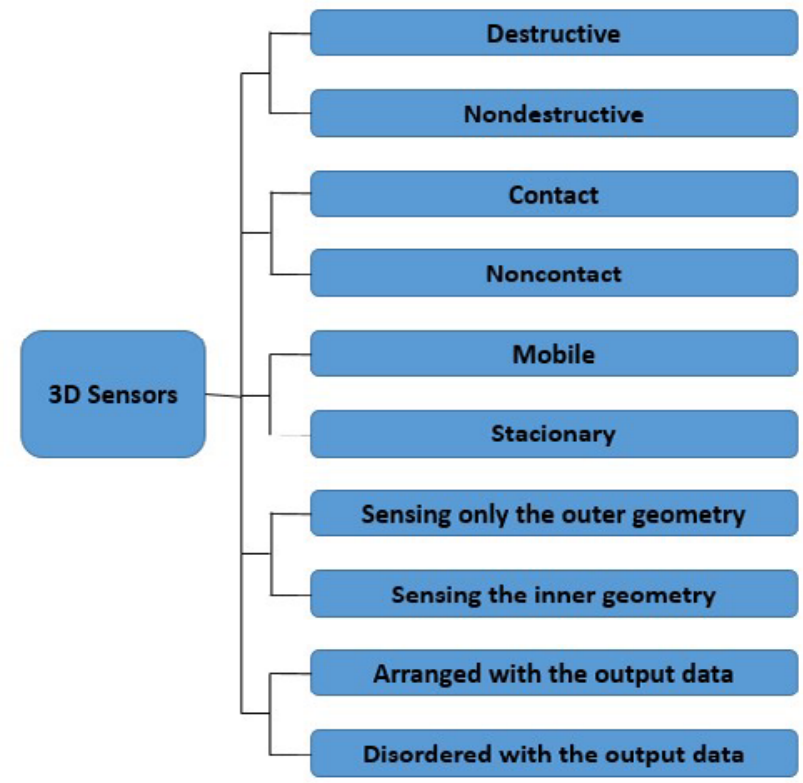

Fig. 7. Distribution of 3D sensors [5].

\section{Principles of sensorial systems}

Acquisition data about a space from a sensor and its processing using some tools is the main purpose of a sensorial system. Sensors providing the important data about a space: 
- $\quad$ Photo sensors - measuring the intensity of a light, or the sensitivity of an optical device.

- Acoustic sensors - measuring of the sensitivity and frequency range of a sound.

- Mechanosensor - mechanical interactions.

- Chemosensors - detecting chemicals.

- Thermo sensors - they use thermistors and other thermoelectric sensors for measuring of the temperature.

Generally, sensorial systems can be divided into two basic groups. There are inner and outer systems. The outer systems provide data about the outer environment and the inner systems provide data only about inner status and parts of a robot. The following actions take place during scanning:

- Localization of objects,

- Studying properties of objects,

- Studying properties of the image of an environment,

- Measuring status of accumulators,

- Orientation in an environment.

\section{Principles of 3D scanning}

3D sensors are coming to the forefront and are more and more commonly used in all possible sectors because of the development of technology and the increasing demand and popularity of virtual reality. Some examples include construction, architecture, industrial machinery, navigation, etc. Perhaps in all applications, it is necessary to have coordinates in a $3 \mathrm{D}$ space. The scanning is dependent on the position of the object, its speed, colour, shape, and angle of rotation. The advent of modern technology and optics, which is still used for 2D scanning, expanded to methods for measuring the third dimension. Today, there are mainly two methods of measuring. The first one is a triangulation and the second is a light interference. The gradual development of technologies brings about the third method. This method is called Time of Light (TOF) and uses the knowledge of the speed of light.

\subsection{Triangulation}

Triangulation is very often used as a method which requires a very complicated structure of the measuring sensor. It is divided into two categories: active triangulation and passive triangulation.

The principle of the active triangulation involves photogrammetric reconstruction of the scanned object. The surface of scanned object is illuminated by the light source and simultaneously scanned by the CCD sensor [7].

The principle of passive triangulation involves photogrammetric reconstruction of the scanned object on the basis of its projection on the sensor surface device. One dimension is lost during projection and it is needed to renew on the basis of a common information from multiple sources [7].

The light signal is transmitted from the laser source to the object. The object reflects the light ray to the camera. The angle of the transmitted ray from the source is constant, but the CCD sensor depending on the spend ray of the dimmed sensor. The connector between the light source and the CCD sensor is called triangulation base (baseline). Thanks to the knowledge of two angles and the length of the triangular base, we can calculate the distance of a point, and then save the coordinates for later calculations and rendering [5].

To mark the surface, the following are used:

- The light beam (1D triangulation),

- Light (2D triangulation),

- $\quad$ Structured light beam (3D triangulation).

Disadvantages:

- Higher purchase price and better facilities,

- Time demands when evaluating the Record,

- The limiting factor may be the memory size and especially the quality of the recording,

- More necessary knowledge of issues,

- Treatment of subjects.

\subsection{Interferometry}

Interferometry is a method suitable for very precise measurements over a short distance. The principle is based on light interference. The principle can be seen in Figure 6. The light source - the laser - is transmitted through the polarization splitter - a part on the measured object. The reflected signal is combined with polarization ray splitter reference called carrier wave and they may interfere together. The resulting wave interference is given by equation (1) [5]:

$$
I(x, y)=\left.I_{1}(x, y)\right|^{2}+\left|I_{2}(x, y)\right|^{2}+2 I_{1}(x, y)|| I_{2}(x, y) \mid \operatorname{co}\left(g_{1}(x, y)-j_{2}(x, y)\right)
$$

\section{Disadvantages:}

- Technologically intensive production,

- Demanding quantitative interpretation of results,

- Susceptibility to interference.

\subsection{Time of light}

This is a method that uses the knowledge of the speed of modulated light signal that is emitted from the transmitter and subsequently reflected towards the receiver. The use of this method requires very precise time value. The principle can be seen in Fig. 7. The distance of sensing object can be computed from formula 2 , where " $t$ " is the total time from sending a signal to the one more acceptation and " $c$ " is the speed of light (c = $2.998 * 108 \mathrm{~m} / \mathrm{s}$ ) [5].

$$
s=\frac{c^{*} t}{2}
$$

Disadvantages:

- High-accuracy time measurement required,

- Measurement of a light pulse return is inexact, due to light scattering.

- Difficulty to generate short light pulses with fast rise and fall times. 


\section{Utilization of triangulation in a real project}

The trajectory of a laser ray (using a laser module M18B532-X-GL, for instance) impacts on objects in a space what is caught by a camera subsystem what is one of the main functional groups of a robot. A robot can contain a colour digital camera (like Go Pro Hero4) with a CMOS sensor positioned in a water and vapour resistant box or coating. The robot could be controlled remotely using a radio frequency $2.4 \mathrm{GHz}$ using approved transmitter and receiver for common outdoor/indoor utilization. The communication will be done using a software programmed (in $\mathrm{CH}$, for instance) providing computations and communication between the robot and an operator.

\section{Conclusion}

The qualities of 3D sensors became higher thanks to the progress in the modern trends in robotics. Grow in this area increases due to better digitalization, cartography, security and other aspects.

The main goal of this work lies in outlining definitions of basic terms and principles of individual categories of sensors. The authors were focused on global aspects and coping whole systems as complex units. Therefore principles of 3D sensors were described without detailed aspects of low-level technologies. Alternatives of sensors and their further utilization in real life were discussed in this work.

However, the progress in application of 3D sensors and $3 \mathrm{D}$ technologies generally is expected to grow more than people usually consider. This statement is based on the research data presented in this paper. The utilization of all the three dimensions (which used to be in seclusion to date) is the main advantage of 3D sensors. The authors guess that traditional 2D sensors will almost entirely vanish and will be replaced with $3 \mathrm{D}$ sensors in several years. New sensors will be more expensive but they will worth the money due to their possibilities in the area of visualisation and robotics.

\section{Acknowledgment}

This work was supported by the Ministry of Education, Youth and Sports of the Czech Republic within the National Sustainability Programme project No. LO1303 (MSMT-7778/2014) and also by the European Regional Development Fund under the project CEBIA-Tech No. CZ.1.05/2.1.00/03.0089. This work was also supported by Internal Grant Agency of Tomas Bata University under the project No. IGA/FAI/2017/004.

\section{References}

[1] L. Kárník, Practical applications of service robots (2011)

[2] J. Bartušek, Mobile device for movement in dangerous environment (2015)

[3] Ch. Winkler, Design Technology [online] (2016)

[4] Sensors \& Transducers, 206 (2016)

[5] M. Marcaník, J. Dvorak, Use of 3D sensors for the protection of critical infrastructure elements and soft targets (2015)

[6] H. Foell, MEMS Sensors and Actuators [online]. (2011)

[7] S.C. Islam, M. Herrmann, R. Beigang, IRMMWTHz2007 (2007)

[8] P. Skoupý, 3D optical measurement and scanning systems for engineering (2007)

[9] F. Brunet, Contributions to Parametric Image Registration and 3D Surface Reconstruction (2010)

[10] W. Schroeder, K. Martin, B. Lorensen, Visualization Toolkit: An Object-Oriented Approach to 3D Graphics (2006)

[11] M. Janková, J. Dvořák, Mathematics, Information Technologies and Applied Sciences, (2014) 